\title{
Criminologie
}

\section{Les voleurs à main armée adultes devant les tribunaux : étude du cheminement des plaintes}

\section{Rosette Gagnon et Marc LeBlanc}

Volume 18, numéro 2, 1985

Le vol à main armée à Montréal

URI : https://id.erudit.org/iderudit/017217ar

DOI : https://doi.org/10.7202/017217ar

Aller au sommaire du numéro

Éditeur(s)

Les Presses de l'Université de Montréal

ISSN

0316-0041 (imprimé)

1492-1367 (numérique)

Découvrir la revue

Citer cet article

Gagnon, R. \& LeBlanc, M. (1985). Les voleurs à main armée adultes devant les tribunaux : étude du cheminement des plaintes. Criminologie, 18(2), 63-83.

https://doi.org/10.7202/017217ar
Résumé de l'article

This article on the channeling of charges through the justice system deals with the punishment of particular infraction, armed robbery, in a comprehensive way, that is, its passage through all aspects of the justice system. To do this a sample of 1258 armed robberies in Montreal and Quebec were used.

The most interesting result of the analysis presented is the finding that the least violent armed robberies give rise to the most exacting police reaction and the most severe judicial treatment. There is a sort of schism between the crime and the reaction it generates. 


\section{LES VOLEURS À MAIN ARMÉE ADULTES DEVANT LES TRIBUNAUX : ÉTUDE DU CHEMINEMENT DES PLAINTES* Rosette Gagnon** Marc Leblanc***}

This article on the channeling of charges through the justice system deals with the punishment of particular infraction, armed robbery, in a comprehensive way, that is, its passage through all aspects of the justice system. To do this a sample of 1258 armed robberies in Montreal and Quebec were used.

The most interesting result of the analysis presented is the finding that the least violent armed robberies give rise to the most exacting police reaction and the most severe judicial treatment. There is a sort of schism between the crime and the reaction it generates.

Une étude sur le vol à main armée a été réalisée à partir des dossiers des corps de police des villes de Québec et de Montréal (Gagnon et LeBlanc, 1984a). Plusieurs aspects ont été traités dont la description et l'analyse des événements de vol à main armée (Gagnon et LeBlanc, 1985), l'enquête policière et la détection des suspects (Gagnon et LeBlanc, 1984b), la poursuite des présumés auteurs devant les tribunaux ainsi que les sentences imposées aux coupables. Pour les infracteurs âgés de moins de 18 ans, les données judiciaires furent compilées au tribunal de la jeunesse après consultation et analyse des informations pertinentes à la Direction de la protection de la jeunesse (LeBlanc, 1985).

L'étude s'est fixée, outre des objectifs descriptifs du phénomène vol à main armée et des interventions policières et judiciaires, des objectifs compréhensifs et dynamiques. Plus exactement, le matériel recueilli avait pour but l'étude du cheminement des plaintes dans le système de justice, c'est-à-dire la mise en relation des informations à chacune des étapes du système pénal.

- Cette recherche a été réalisée grâce à une subvention triennale de la Fondation F.C.A.R. et de l'Université de Montréal.

** Criminologue, Centre international de criminologie comparée.

*** Professeur titulaire, École de criminologie et chercheur associé, Centre international de criminologie comparée, Université de Montréal. 


\section{L'ÉCHANTILLON}

L'étude a porté sur $1258^{1}$ vols à main armée, c'est-à-dire des vols qualifiés commis avec une arme offensive ${ }^{2}$. Ces vols ont été rapportés aux corps municipaux de Québec pour les années 1979 et 1980 et à la police de la communauté urbaine de Montréal au cours de janvier 1980. L'échantillonnage a été fait au hasard et les proportions retenues ont été différentes selon que les vols étaient solutionnés ou non; cette façon de faire avait pour but de nous assurer d'un nombre suffisamment élevé de suspects poursuivis devant les tribunaux. Ainsi, nous avons sélectionné 705 événements solutionnés et 561 non solutionnés.

\section{LES TYPES DE VOLS À MAIN ARMÉE}

Une étude descriptive détaillée des 1258 vols à main armée nous a amenés à constater que cette forme de conduite criminelle est diversifiée; qu'en dépit de son homogénéité apparente, elle recèle des manifestations variées.

Les types de vol à main armée ont été constitués à partir de deux variables clés, à savoir la cible du vol et le nombre de suspects. Dans une première étape, dix types de vols à main armée ont été élaborés et étudiés de manière comparative en fonction des variables descriptives qui se sont avérées les plus importantes dans l'examen de cette forme de criminalité. Ces variables, au nombre de treize, sont les suivantes : nature du vol (arme à feu ou autre), montant du vol, âge des suspects, recours à un déguisement et nature de ce dernier, recours à la violence (utilisation effective des armes, violence physique), attitude des témoins et/ou victimes (résistance, intervention pour arrêter les suspects), nombre de témoins, utilisation d'un véhicule et observation du moyen de fuite des voleurs. L'analyse nous a conduit à identifier six types de vols suffisamment différents les uns des autres pour justifier de les considérer séparément.

Les vols commis en groupe contre les institutions financières (type 1) sont toujours commis avec une arme à feu $(93,3 \%)$ et le montant du vol est le plus élevé de tous les types avec une

1. À l'origine 1266 vols à main armée ont été étudiés mais l'analyse a porté sur 1258 vu le manque d'informations sur huit de ces événements.

2. Précisons que c'est le vol qualifié qui constitue un acte criminel, le vol à main armée étant compris dans l'article 302 du code criminel. 
médiane de 2350 . Les voleurs ont en moyenne 21,1 ans et c'est pour ces vols où ils sont le plus souvent déguisés $(56,5 \%)$. On observe que c'est le type de vol où il y a le moins souvent de violence et où il y a peu ou pas de résistance de la part des victimes (moins de $10 \%$ des cas). Par ailleurs, on note que dans plus de la moitié des cas $(53,2 \%)$ un véhicule est utilisé pour assurer la fuite des voleurs. Soulignons que dans plus des deux tiers des cas, il y a trois témoins ou davantage. Les vols commis seul contre ces mêmes institutions financières (type 2) sont encore commis avec une arme à feu $(97 \%)$, et le montant du vol est le deuxième en importance, la médiane étant de $1452 \$$. À la différence du type précédent, le voleur est nettement plus âgé, ayant 25,1 ans en moyenne. Le recours au déguisement est beaucoup plus rare $(28,4 \%)$ et on note que c'est le type de vol pour lequel la violence est à son plus bas. Le vol commis par une personne seule contre une telle cible se caractérise essentiellement par sa discrétion, plusieurs témoins ne réalisant même pas qu'il y a vol, cela contrairement au type précédent où le groupe sert de moyen d'intimidation. Les scénarios d'exécution du vol sont donc très différents pour ces deux types de vol à main armée.

Les vols commis en groupe contre les commerces intermédiaires (type 3) (pharmacies, épiceries, restaurants, bijouteries, S.A.Q., grands magasins...) le sont avec une arme à feu dans un peu plus de $80 \%$ des cas; le montant du vol est nettement inférieur aux types précédents avec une médiane de $500,00 \$$ alors que l'âge des suspects se compare au premier type ( 21,5 ans en moyenne). Le recours au déguisement survient pour le quart des suspects et un véhicule est utilisé dans un plus du tiers des cas. La violence est notée dans près de $35 \%$ des cas ce qui place ce groupe de vols à main armée au second rang à ce chapitre précédé des vols commis contre les particuliers. $\grave{A}$ la différence des institutions financières, les commerçants sont ici directement impliqués dans les pertes relatives au vol et c'est probablement la raison pour laquelle les victimes interviennent pour protéger leurs biens rendant ainsi l'exécution du délit plus difficile et les manifestations de violence plus fréquentes. Les vols commis seul contre les mêmes commerces (type 4) sont le fait d'un suspect plus âgé (23,9 ans) et ayant davantage recours à une arme offensive autre qu'une arme à feu $(33,8 \%)$, généralement un couteau. Dans près de $50 \%$ des cas, le voleur n'est confronté qu'avec la victime, ce qui rend le contrôle plus facile que dans les autres types. 
Les vols commis contre les magasins de variétés, dépanneurs, tabagies et gaz-bar (type 5) sont numériquement les plus importants; qu'ils soient commis seul ou en groupe, leurs caractéristiques sont fort comparables et c'est pourquoi nous n'en formons qu'un seul type. Ce vol est commis avec une arme offensive autre qu'une arme à feu sensiblement dans les mêmes proportions que le type précédent $(34,6 \%)$ ce qui traduit le côté moins «professionnel» ou moins organisé des voleurs. Le montant du vol est le plus faible (médiane de 140,00\$) et moins du cinquième des voleurs sont déguisés. On ne peut pas dire que c'est le type de vol où la violence est particulièrement fréquente $(21,5 \%)$ et cela se comprend du fait que dans plus de $60 \%$ de ces vols, il n'y a aucun témoin sauf la victime. La situation même dans laquelle se retrouve la victime la force en quelque sorte à consentir au vol. On pourrait dire que c'est un vol facile mais peu payant; il peut donc être commis par des voleurs moins expérimentés d'autant plus qu'il nécessite très peu de préparation.

En dernier lieu, il y a les vols à main armée commis contre des particuliers (chauffeurs de taxi, préposés de stationnement, personnes sur la rue... (type 6). On note que dans $52 \%$ de ces vols, proportion la plus élevée à ce chapitre, le vol est commis avec une arme autre qu'une arme offensive (couteau, bâton, instrument contondant, ...). C'est également le type de vol à main armée le plus violent $(57,6 \%)$ et cela vraisemblablement pour deux raisons : la nature de l'arme et le contact avec la victime, l'argent se trouvant souvent sur elle. D'ailleurs dans $31 \%$ des cas, on observe que la victime immédiate oppose une résistance physique. Elle est plus touchée par l'éventuelle perte monétaire. C'est un vol très violent bien que moins payant (médiane $150,00 \$$ ); à la différence du type précédent, il est plus dangereux vu les sortes d'armes utilisées et la nécessité du contact avec la victime. Précisons, en dernier lieu, que dans plus de $75 \%$ des cas, il n'y a aucun témoin.

Ces types de vols à main armée se distinguent par leur degré d'organisation et de préparation : être capable de se procurer une arme à feu par opposition à une arme de fortune, rendre sa description et par conséquent son identification, plus difficile par le recours à un déguisement, choisir une cible potentiellement payante, éviter le plus possible l'utilisation de violence, utiliser un véhicule pour faciliter sa fuite... Peut-on penser que ces types de 
vol de par leurs caractéristiques respectives, déterminent des réactions pénales différentes?

\section{CRIME NON HOMOGÈNE, MODE DE RÉSOLUTION NON HOMOGÈNE}

Notre étude du cheminement des plaintes dans le système de justice nous a permis de mettre en évidence un certain nombre d'éléments interdépendants et c'est pourquoi il importe d'apporter des précisions quant aux modes de résolution des vols à main armée avant de procéder à l'analyse de la réaction des tribunaux. Cette dernière n'aurait pas de sens, croyons-nous, si nous ne tenions d'abord compte de l'intervention policière.

Dans un premier temps, nous observons que, toutes proportions gardées, les six types de vols à main armée n'affichent pas des taux de solution comparables ${ }^{3}$. Les vols commis contre les institutions financières donnent lieu plus souvent que les autres à des poursuites judiciaires. Ces pourcentages sont respectivement du type un au type six de : 74,5, 69,3, 51,7, 45,4,53,1 et 54,8. Précisons que ces proportions résultent de l'échantillonnage mais ne doivent pas être confondues avec les statistiques officielles'. Une étude détaillée du moment où surviennent les arrestations nous permet de voir que, si les taux de solution sont différents, les modes de résolution le sont également. Plus exactement, nous observons qu'à mesure que diminuent les taux de solution augmente la proportion des arrestations à survenir le jour même du délit. Ces vols commis en groupe contre les institutions financières sont ceux où le taux de solution est le plus élevé mais également ceux où une arrestation survient rarement le jour même du délit. Concrètement, on constate que les vols dirigés contre les institutions financières par plus d'un suspect constituent le type pour lequel la solution

3. Un vol est considéré comme solutionné à partir du moment où au moins une personne est poursuivie par voie de mise en accusation.

4. Les techniques d'échantillonnage ont volontairement sélectionné davantage de cas solutionnés qu'il n'en existe en réalité, les taux de solution précis du vol à main armée étant de plus ou moins $20 \%$. 
des cas est la plus élevée alors qu'au même moment, c'est le type où l'arrestation d'au moins un suspect survient rarement le jour même du délit, il s'écoule au moins une semaine pour $69,6 \%$ des arrestations. Les vols commis seuls contre les mêmes institutions viennent en deuxième lieu quant à la proportion des cas solutionnés bien que dans $47,4 \%$ des cas, l'arrestation survienne après la première semaine. Pour les vols de commerces intermédiaires, en groupe, le taux de solutions est inférieur aux types précédents mais les arrestations se font plus rapidement (médiane de 4,2 jours). Pour cette même cible mais dont le vol est le fait d'une seule personne, le taux de solution est le plus faible de tous les types mais les arrestations le jour même sont parmi les plus fréquentes $(44,2 \%)$. Concernant les magasins de variétés et les dépanneurs, le taux de solution est moyen en comparaison à l'ensemble et le taux d'arrestation au cours de la première semaine se compare aux types précédents. Enfin, pour les particuliers, le tatx de solution est moyen mais près de $80 \%$ des arrestations ont lieu la première semaine alors que près de $50 \%$ surviennent le jour même (tableau 1).

Les éléments sous-jacents à la résolution d'un cas, et nos travaux ont fait ressortir des différences selon les types, peuvent tenir essentiellement à la nature du délit, l'attitude des témoins et victimes, la réaction policière au niveau des patrouilleurs et enfin l'enquête ultérieure. Dépendant des types de vols à main armée, les possibilités de résolution varient en fonction de la prédominance de certains facteurs liés aux délits : planification, organisation, assistance technique, nombre de voleurs, etc. Une autre variable est apparue déterminante : la poursuite de l'enquête policière par une escouade spécialisée. Dans le cas des vols d'institutions financières nous avons observé que les arrestations surviennent plus tardivement que pour tous les autres types : le fait est que l'enquête ne cesse pas avec la prise de rapport mais se poursuit par des études de modus operandi notamment. Les taux de solution plus élevés pour les vols d'institutions financières ne s'expliquent donc pas par une moins bonne exécution des délits (au contraire, il y a peu de flagrant délit) mais plutôt par une enquête policière plus poussée. On peut donc dire qu'il y a des types de vols à main armée pour lesquels le fait de ne pas être arrêté tôt après le délit est en quelque sorte une garantie de ne pas être relié au vol alors que dans le cas des institutions financières, le temps joue en quelque sorte contre soi. 
TABLEAU 1

Délai des arrestations selon les types de vols d̀ main armée

\begin{tabular}{|c|c|c|c|c|c|c|c|}
\hline Délais & Types & $\begin{array}{l}\text { Institutions } \\
\text { financières } \\
\text { groupe }\end{array}$ & $\begin{array}{c}\text { Institutions } \\
\text { financières } \\
\text { seul }\end{array}$ & $\begin{array}{c}\text { Commerces } \\
\text { inter. } \\
\text { groupe }\end{array}$ & $\begin{array}{l}\text { Commerces } \\
\text { inter. } \\
\text { seul }\end{array}$ & $\begin{array}{c}\text { Magasins } \\
\text { de variétés, } \\
\text { dépanneurs } \\
\text { groupe et seul }\end{array}$ & $\begin{array}{l}\text { Particuliers } \\
\text { groupe } \\
\text { et seul }\end{array}$ \\
\hline Ensemble & Le jour même & 15,4 & 37,3 & 35,9 & 44,2 & 36,1 & 47,6 \\
\hline des accusés & $2 e-7 e$ jr. incl. & 14,9 & 15,2 & 21,5 & 21,4 & 29,6 & 31,9 \\
\hline$(\mathrm{N}-1 \mathrm{176})$ & ge jr. ou plus & 69,6 & 47,4 & 42,5 & 34,3 & 34,2 & 20,5 \\
\hline Premier & Le jour même & 16,9 & 35,8 & 36,7 & 50,8 & 36,4 & 47,0 \\
\hline accusé & $2 e-7 e \mathrm{jr}$. incl. & 15,1 & 13,2 & 20,5 & 21,3 & 29,8 & 30,4 \\
\hline seulement & $8 \mathrm{e}$ jr. ou plus & 67,9 & 50,9 & 42,8 & 27,8 & 33,8 & 22,5 \\
\hline$(N=701)$ & Médiane & 18,5 & 9,7 & 4,2 & 0,5 & 2,4 & 0,8 \\
\hline
\end{tabular}




\section{ENQUÊTE POLICIÈRE, TYPES DE VOLS À MAIN ARMÉE ET QUALIFICATION LÉGALES : UN LIEN ÉTROIT}

Les observations faites précédemment ne sont pas sans rapport avec le sort réservé aux voleurs à main armée. Pour l'ensemble des 1163 personnes mises en accusation ${ }^{5}$ par la police on observe que, quantitativement parlant, le nombre de chefs d'accusation varie en fonction des types de vols à main armée. Dans le cas des vols commis en groupe contre les institutions financières, ce n'est que pour $3,2 \%$ des accusés qu'il n'y a qu'un seul chef; dans tous les autres cas, le chef principal est assorti de chefs secondaires. Pour ces mêmes cibles, il y a trois chefs ou davantage pour $55 \%$ des accusés, cette proportion étant respectivement des types deux à six de $7,6 \%, 29,7 \% 16,3 \%, 15,8 \%$ et $21,3 \%$. Il va de soi que les vols commis en groupe peuvent entraîner une poursuite en vertu du complot mais il peut également y avoir d'autres chefs accessoires. Qualitativement parlant cette fois-ci, l'article 309.2 concernant le déguisement est allégué contre $43,8 \%$ des accusés du premier type de vol, ce qui constitue de loin la proportion la plus élevée. On se souviendra que les voleurs sont le plus souvent déguisés dans les vols commis en groupe contre les institutions financières. Mentionnons aussi que les personnes accusées de vols commis seul contre les commerces intermédiaires, seul et en groupe, contre les magasins de variétés et les dépanneurs sont le moins souvent poursuivis pour déguisement $(9,3 \%, 8,1 \%$ et $5,1 \%)$. L'utilisation d'une arme à feu [article 83 (1)] est mentionnée pour $16,6 \%$ de l'ensemble des accusés ${ }^{6}$. Cette proportion est équivalente selon les types de vols à main armée sauf pour les vols commis contre les particuliers où la proportion d'une telle poursuite est de $5,1 \%$. C'est le type de vol le plus souvent commis avec une autre arme offensive que l'arme à feu. En dernier lieu,

5. La même personne peut ici figurer plus d'une fois étant impliquée dans plus d'un vol à main armée; il ne s'agit donc pas forcément de personnes différentes.

6. Pour poursuivre en vertu d'un article 83 , la jurisprudence s'accorde pour dire que l'arme doit être produite en pièce à conviction pour en établir sa qualification légale. Précisons par ailleurs que l'article 83(1) commande une peine minimum d'un an (première offense) et consécutive à toute autre peine d'où sa très grande importance dans une dénonciation. 
soulignons l'importance du chef de séquestration dans le cas des vols contre les particuliers $(10,3 \%)$ et contre les accusés des vols de commerces intermédiaires commis en groupe $(7,1 \%)$. Les poursuites viennent confirmer le fait que les contacts avec les victimes sont plus fréquents pour les types de vols et que par conséquent la violence est plus manifeste.

Pour terminer cette section des poursuites entamées par les corps policiers, ajoutons qu'au niveau du chef principal nous observons des différences suffisament importantes pour être mentionnées. D'une façon générale, le suspect sera poursuivi en vertu d'un article 302 pour lequel il peut préciser l'alinéa A, B, C ou D. La nuance entre ces qualifications est parfois difficile à établir puisque l'alinéa cherche à apporter une précision sur la nature du vol qualifié. Généralement, lorsqu'on parle de vols à main armée on fait allusion à la catégorie $302 \mathrm{D}$; l'article $302 \mathrm{C}$ définit la tentative à l'intérieur du vol qualifié ce qui a pour effet une peine supérieure aux tentatives définies à l'article $421^{7}$. À l'alinéa $\mathrm{A}$ il est question de violence ou de menace de violence alors que l'alinéa B traite des cas où le suspect «blesse, bat ou frappe» la victime. Entre les types de vols à main armée, le pourcentage des accusés poursuivis pour le chef principal, article $302 \mathrm{D}$ est le type un à six, respectivement de : $89,1 \%, 65,7 \%, 63,3 \%, 72,9 \%$, $64,8 \%$ et $57,7 \%$. Bien qu'en principe ces distinctions sont d'une importance secondaire puisque l'article 303 prévoit une même peine pour chacun de ces alinéas, on verra plus loin certaines différences. Précisons que pour $15,9 \%$ des accusés, les policiers se sont contentés d'inscrire l'article 302 à leur plainte; à cela ne correspond aucune infraction et au niveau du tribunal la précision devra être apportée. Cette difficulté à identifier l'alinéa toutefois rend compte de l'ambiguïté de certains vols à main armée. Les tentatives sont rares mais elles sont les plus fréquentes pour les vols commis contre les particuliers $(8,1 \%)$.

Les différences observées au niveau des chefs principaux et secondaires font état de différences ou du moins de nuances dans

7. Dans le cas d'une tentative d'acte criminel passible de la perpétuité, le coupable est passible de 14 ans d'emprisonnement (article 421.1). Dans le cas d'une tentative de vol qualifié, article $302 \mathrm{C}$, il est passible de la peine prévue à l'article 303 , soit la perpétuité. Cette particularité de la peine pour tentative est comparable à celle prévue pour la tentative de meurtre, article 222. L'intention du législateur serait donc une plus grande sévérité pour ces tentatives. 
la réalisation des vols à main armée, présentant par là un crime non homogène dans son déroulement d'une part et dans certains autres cas d'investigations policières différentes.

Nos données ont fait ressortir que les qualifications légales retenues par les corps policiers étaient généralement maintenues par les procureurs d'où une concordance des chefs presque parfaite. Précisons que les articles 302 sans précision d'alinéa deviennent pour la très grande majorité des articles $302 \mathrm{D}$. Cette première analyse fait ressortir que les voleurs à main armée ne se présentent pas devant les tribunaux avec des accusations identiques et ces motifs sont variables selon les types.

\section{LE TRAITEMENT JUDICIAIRE DES VOLEURS À MAIN ARMÉE : POINTS SAILLANTS}

Notre étude au niveau des tribunaux adultes a porté sur $\mathbf{5 1 5}$ individus différents poursuivis pour au moins un vol à main armée de notre échantillon au niveau policier. Notre analyse a porté sur le contenu des dénonciations, sur les procédures pénales et enfin sur les verdicts et sentences.

Dans un premier temps précisons qu'un individu peut être poursuivi pour plus d'un délit à la fois dans la mesure où plusieurs infractions sont élucidées en même temps par un corps policier. Ceci revient à dire qu'une même dénonciation peut contenir plusieurs événements : dans de tels cas il n'est plus possible d'associer la sentence à un événement en particulier parce que le juge doit tenir compte de l'ensemble des chefs sur lesquels l'accusé plaide ou est reconnu coupable. Pour ces cas, il n'est donc pas possible de faire l'étude des sentences relatives au vol à main armée seulement. Partant de là deux formes d'analyse ont été conduites pour procéder à l'étude des sentences : celle par rapport au contenu de l'ensemble de la dénonciation et celle pour les vols à main armée avec référence à la typologie, c'est-à-dire les seuls cas ne comportant dans la dénonciation qu'un vol à main armée.

\subsection{CONTENU DES DÉNONCIATIONS}

Près des deux tiers des individus poursuivis dans le cadre de notre échantillon le sont pour un seul événement, c'est-à-dire le vol à main armée retenu dans notre étude $(64,3 \%) ; 13,8 \%$ le 
sont pour deux délits et enfin, proportion non négligeable, $21,9 \%$ le sont pour trois infractions ou davantage (moyenne : 2,10, médiane 1,28$)$. Le nombre des événements rapportés dans la dénonciation fluctue de manière fort appréciable selon les types de vols à main armée; l'individu ayant commis un vol contre une institution financière avec complice et lorsque pris pour ce vol, est dénoncé le plus souvent pour d'autres délits que celui-là. Cela revient à dire que l'enquête policière a réussi à relier plusieurs délits à un même suspect, ce qui permet de penser que l'enquête a porté non seulement sur un événement à solutionner mais également sur un individu à poursuivre. Du type un au type six, les proportions de cas où un seul événement est reproché dans la dénonciation sont les suivantes : $36,1,43,6,72,9,72,9,63,7$, et 76,4 . Cette observation est d'une très grande importance puisqu'elle met en lumière des enquêtes policières différentes et par conséquent, probablement des traitements judiciaires différents en raison de dossiers plus chargés.

Cette donnée montre la nécessité d'une étude du cheminement des plaintes dans le système de justice en raison du lien étroit entre le travail des policiers et la poursuite pénale.

\subsection{PROCÉDURES PÉNALES}

Le délai qui s'écoule entre l'arrestation d'un suspect et la première comparution confirme la règle voulant que le prévenudétenu soit amené devant un juge dans les vingt-quatre heures suivant sa mise aux cellules. La moyenne de ce délai est de 6,31 jours et la médiane de 1,30 jours. Ceci étant dit, ajoutons que pour les suspects non détenus, le délai entre l'arrestation et la comparution est de 60,4 jours en moyenne avec une médiane de 48,0 $(\mathrm{N}=19)$. Il s'agit là de suspects ayant comparu sur sommation, ce qui représente une minorité d'individus.

Puisque plus de $95 \%(493 / 515)$ des prévenus sont détenus lors de leur première comparution, il va de soi que pour plusieurs d'entre eux la première préoccupation consistera à obtenir une remise en liberté pour la durée des procédures précédant le verdict. On note que le cautionnement est accordé à $39,2 \%$ de ceux qui en font la demande; $71,6 \%$ de ceux à qui il est accordé n'ont qu'un seul événement reproché dans la dénonciation contre 55,2\% pour ceux à qui il est refusé. La moyenne d'événements au dossier fait ressortir qu'il y en a 2,62 dans les cas de refus et 1,49 dans 
les cas d'acceptation. D'un strict point de vue quantitatif, l'octroi du cautionnement est fonction du contenu de la dénonciation. Par ailleurs, on observe que la présence d'un article 83 (1) (utilisation d'arme à feu) compromet largement les chances d'un cautionnement puisqu'il n'est accordé qu'à $19,8 \%$ des infracteurs poursuivis pour le chef contre $43,9 \%$ pour ceux qui n'en sont pas accusés. Quant aux chefs de complot et de déguisement, les différences sont moins marquées.

C'est la variable antécédents qui est la plus déterminante au niveau du cautionnement : il est accordé à $26,4 \%$ de ceux qui en ont et à $51,3 \%$ de ceux qui n'en affichent pas ${ }^{8}$. D'une autre façon on observe que $40 \%$ de ceux à qui il est accordé ont déjà une condamnation antérieure contre $66 \%$ de ceux à qui il est refusé. $\mathrm{Si}$ le nombre et la nature des événements impliqués dans la dénonciation ont un rapport avec le cautionnement il ressort que c'est la présence ou l'absence d'antécédents chez l'inculpé qui paraît être le facteur le plus déterminant.

L'enquête préliminaire vise à déterminer s'il y a matière à procès; elle aura lieu dans les cas où le prévenu choisit d'être jugé par un juge et un jury ou par un juge seul; s'il opte pour un magistrat, il sera directement mis en accusation. Nos données révèlent qu'entre le moment de la première comparution et celui où le prévenu est renvoyé pour son enquête préliminaire, il y a un délai médian de 10,6 jours avec un mode de sept jours. Lorsque l'individu est détenu, ce délai ne doit pas excéder huit jours francs [article 465 (1) b] à moins qu'il n'y consente ou qu'il soit envoyé pour examen. Ces prescriptions sont donc observées. Pour les $\mathbf{4 7 2}$ prévenus pour lesquels une enquête préliminaire aurait pu avoir lieu, on note que $32,4 \%$ d'entre eux y ont renoncé en conformité avec les dispositions de l'article 476 (1).

\subsection{LES PLAIDOYERS ET VERDICTS}

À n'importe quel moment, au cours du déroulement des procédures, l'accusé peut, s'il le désire, enregistrer un plaidoyer de culpabilité plutôt que de subir un procès. On imagine facilement

8. Il est ici question d'antécédents officiels, c'est-à-dire de la présence de condamnations antérieures devant les tribunaux. 
que l'individu qui plaide coupable accélère le processus judiciaire puisque après un tel plaidoyer il ne reste au juge qu'à imposer une sentence. Les plaidoyers toutefois portent sur chacun des chefs compris dans la dénonciation; il peut donc $\mathrm{y}$ avoir des cas où l'inculpé plaide coupable sur un ou plusieurs chefs mais non sur tous, ce qui nous force à revoir l'ensemble du dossier pour mieux comprendre les relations entre verdict et sentence.

D'abord on observe que $87,7 \%$ des inculpés plaident ou sont reconnus coupables sur au moins un chef de la dénonciation originale ce qui constitue une proportion fort importante. Si nous ajoutons les quelques cas où il fut coupable sur une infraction moindre ou incluse ou autre, cette proportion atteindrait $90 \%$. Cette première observation nous fait voir que peu d'accusés échappent totalement à la sanction pénale lorsqu'ils sont traduits en justice.

Une autre constatation intéressante porte sur 22,3\% des dossiers pour lesquels au moins un chef de la dénonciation est retiré; cela signifie que pour ces cas les plaidoyers sont mixtes (à moins que tous les chefs ne soient retirés). En pratique, cela signifie que l'individu plaide coupable ou est reconnu coupable sur un certain nombre de chefs et qu'au même moment la couronne accepte d'en retirer un ou davantage. Cette pratique est souvent identifiée comme étant la "négociation du plaidoyer» en ce qu'un compromis est négocié entre la peine à purger et les chefs à retirer de la dénonciation. Les négociations toutefois n'ont pas d'impact sur la nature de la peine et sur la durée de l'emprisonnement mais influencent de manière significative la durée du processus pénal.

L'analyse de cette variable, en fonction des types de vols à main armée, fait voir quelques différences intéressantes à souligner, bien qu'il serait exagéré de parler de différences significatives puisqu'il n'y a que deux profils prédominants de plaidoyers lesquels rendent compte de la presque totalité des cas. Pour ce qui est des vols commis contre les institutions financières, en groupe ou seul, aucun accusé n'est acquitté ou libéré sur tous ses chefs, pas plus que tous ses chefs ne sont retirés. Il y a $42,3 \%$ des accusés de ces vols commis en groupe qui plaident coupable sur toute la dénonciation et il en va ainsi pour $70,6 \%$ des voleurs ayant procédé seul. Les personnes accusées de vols commis dans un commerce intermédiaire plaident coupable sur tous leurs chefs dans des proportions de $58,1 \%$ pour les vols commis en groupe 
et $57,1 \%$ pour ceux commis seul. Au même moment, $10,7 \%$ de l'ensemble de ces accusés échappent à la sanction pénale parce qu'ils sont acquittés ou libérés sur tous leurs chefs. Les inculpés de vols commis contre les variétés et dépanneurs ont ceci de caractéristique que dans $72,2 \%$ des cas, ils plaident coupable sur tous les chefs, ce qui représente la proportion la plus élevée à ce chapitre. Enfin, les personnes accusées de vol contre les particuliers ne plaident coupable sur tous les chefs que dans $44 \%$, alors qu'elles sont ainsi reconnues dans $7,1 \%$ (procès); elles sont coupables d'une autre infraction que celle alléguée dans la dénonciation dans $7,1 \%$ des cas. Ajoutons que $10,8 \%$ de ces accusés échappent à la sanction pénale parce que libérés ou acquittés.

Au terme de cette analyse, retenons que plus de la moitié des suspects plaident coupable sur tous les chefs compris dans la dénonciation que près de $90 \%$ des suspects sont coupables sur au moins un chef et enfin que les chefs retirés sont essentiellement des chefs secondaires bien qu'au moins un d'entre eux soit conservé dans l'accusation et jugé coupable sur plaidoyer. Il est donc clair que la décision de plaider coupable sur tous les chefs est fonction pour une bonne part de l'ampleur et de la nature de la dénonciation principalement en ce qui a trait aux chefs secondaires.

\subsection{LES SENTENCES}

Les sentences imposées dans les dossiers comportant au moins un vol à main armée présentent une certaine homogénéité en ceci que pour plus de $80 \%$ d'entre eux une peine d'emprisonnement continu est imposée. Par ailleurs, dans $60 \%$ des cas une seule sorte de peine est prononcée contre l'accusé. Les sentences pour vol à main armée sont peu diversifiées et la durée de l'emprisonnement varie en fonction principalement de nombre d'événements dans la dénonciation du nombre d'articles $302 \mathrm{D}$ pour lesquels l'accusé est coupable ainsi que du nombre d'articles 83 (1) pour lesquels il est également coupable. Du côté des procédures pénales en matière d'option/réoption ainsi que des plaidoyers nous n'observons aucune différence significative quant au choix de la peine. Concernant l'accusé, ce sont l'âge et la présence ou non d'antécédents qui influencent la diversité et la nature de la sentence.

Nous pourrions tirer de ce canevas général que les facteurs pouvant susciter une certaine forme de clémence de la part des 
juges sont : un seul événement dans la dénonciation, absence de complot et d'utilisation d'arme à feu, l'accusé a moins de $\mathbf{2 0}$ ans ou plus de 30 ans et enfin il n'a pas d'antécédents officiels. À l'inverse, l'individu traduit devant la justice pour plus d'un méfait, ayant commis un vol à main armée avec complice, ayant eu recours à une arme à feu [ou plus exactement étant poursuivi pour un article $83(1)$ ], ayant entre 20 et 30 ans avec des antécédents connus, est le candidat type pour une sentence moins diversifiée, comportant dans la presque totalité des cas un emprisonnement continu et dont la durée sera plus élevée que dans les autres cas et devant par conséquent être purgée dans les pénitenciers.

Concernant le déroulement même du vol, nous avons vu au niveau de l'analyse par types que nous ne trouvions aucun lien direct avec la sentence, les quelques relations observées se manifestant dans le sens opposé de nos prévisions. Ces constatations nous ont amené à voir qu'au niveau du tribunal ce sont essentiellement la nature et la quantité des chefs où l'accusé est reconnu coupable qui ont une influence sur la sentence, plus que le déroulement lors $\mathrm{du}$ vol. Les vols pour lesquels la violence était plus manifeste étaient souvent ceux où une arme à feu était moins souvent utilisée et/ou ceux qui ne réussissaient pas. En conséquence à cela, les accusations ne comportaient pas d'article 83(1) et le chef principal était plus souvent la tentative de vol qualifié (302 C ou 421 de 302 A, B, D) ou le vol qualifié lui-même, mais formulé en article $302 \mathrm{~A}$ ou B. Bien qu'en principe la peine prévue à l'article 303 est la même pour toutes les catégories de vol qualifié, il semble que lorsqu'il s'agit d'un article $302 \mathrm{~A}$ ou $302 \mathrm{~B}$ la peine soit moins sévère dans la diversité, dans l'occurrence de la détention et dans la durée de cette dernière.

Ces informations apparaissent intéressantes en ceci que la formulation de l'accusation n'est pas sans conséquence sur le traitement réservé aux coupables. Plus exactement, le vol qui aux yeux des témoins et victimes paraît teinté d'une violence certaine ne correspondra pas forcément à une sévérité plus grande dans la peine en raison de la nature des chefs retenus contre l'accusé. Pourrait-on dire qu'il y a décalage entre la «hiérarchisation» du facteur violence au niveau des articles du code pénal et la violence telle que déployée dans un vol? Si les vols commis avec arme à feu sont généralement moins violents que ceux commis avec une autre arme offensive, quel sens peut avoir l'article 83(1) dans la 


\section{SCHEMA 1}

Les déterminants de la sentence 1

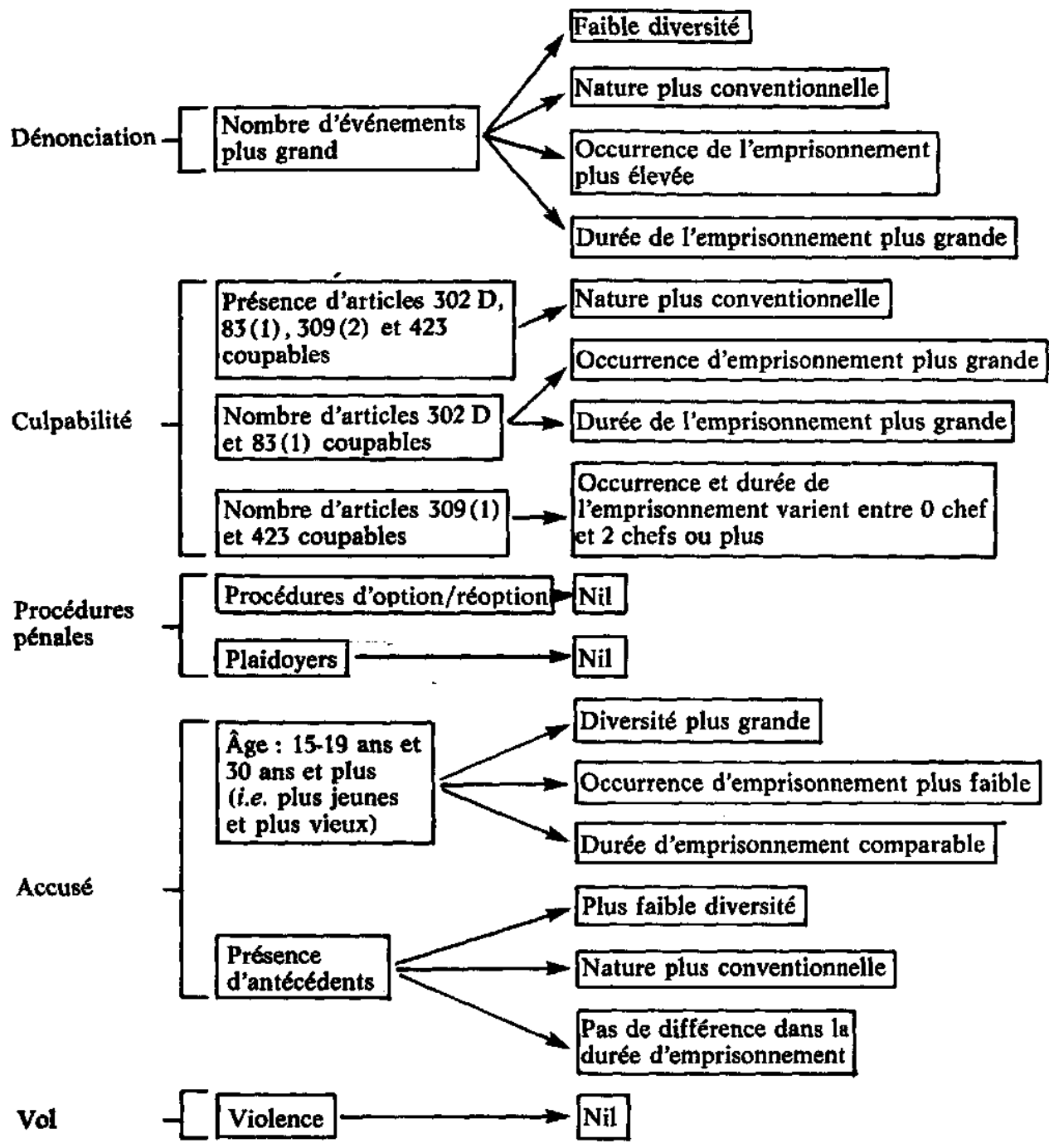

1. La sentence est ici étudiée selon la diversité des types de sentence, la nature de la peine, son occurrence ainsi que la durée de l'emprisonnement lorsqu'il y a lieu. Par diversité, il faut entendre le nombre de types de sentences. Par nature plus conventionnelle, il faut comprendre l'emprisonnement seulement par opposition à l'emprisonnement plus autre chose et à autre mesure sans emprisonnement. 
gradation de la sévérité de la peine? Le facteur quantité d'événements dans la dénonciation paraît être plus important que les caractéristiques propres aux différents vols; dans ce sens on peut dire que lorsqu'un corps policier ou une escouade spécialisée parvient, par son travail d'analyse de modus operandi, à relier divers crimes et à les imputer à un individu, le risque d'une sentence sévère augmente pour ce dernier.

Quelles sont les sentences pour vol à main armée? Retenons qu'elles sont principalement fonction de l'étendue de la poursuite ainsi que des formulations légales. Ces dernières pour leur part doivent tenir compte des éléments de preuve susceptibles de soutenir les allégations et dans ce sens, l'article $83(1)$ est un très bon exemple puisque, en pratique, l'arme doit être retrouvée et produite en pièce à conviction si l'on veut poursuivre l'accusé en vertu de cette disposition. Ces nuances montrent que si certains vols à main armée sont comparables du point de vue de leur exécution, les poursuites peuvent mettre en évidence des crimes fort différents ou du moins appelant des sentences assez diffé. rentes. Les vols à main armée commis contre les particuliers illustrent bien ce fait : le déroulement figure parmi les plus violents et la peine est la moins sévère. À l'opposé, les vols commis en groupe et seul contre les institutions financières font état en général d'une moins grande violence mais rendent compte des sentences les plus sévères. Dans les deux cas, les qualifications légales retenues ainsi que la possibilité d'alléguer certains chefs secondaires rendent compte de ces différences.

\section{RÉACTION DES SYSTÈMES POLICIERS ET PÉNAL AU VOL À MAIN ARMÉE : UNE AMBIGUÏTÉ}

Les données font voir que les différents types de vol à main armée ne se déroulent pas avec la même intensité de violence, qu'ils déterminent des qualifications légales qui varient en fonction des types de vol ( $302 \mathrm{~A}, \mathrm{~B}, \mathrm{C}$ ou D) et qu'enfin, les sentences associées à ces qualifications légales ne sont pas toujours comparables. Également, nous avons vu que l'article 83 n'est pas allégué dans tous les cas impliquant une arme à feu en raison des difficultés à établir les preuves (du moins la qualification légale de ladite arme). Plus qualitativement parlant, nous avons observé une relation inverse entre la violence et la sentence en ceci que les types de vol à main armée où les manifestations de violence sont 
les plus marquées sont aussi ceux où la qualification légale est le moins souvent le vol qualifié au terme de l'article $302 \mathrm{D}$ mais plutôt aux termes des alinéas A et $\mathrm{B}$ dudit article. Par ailleurs, ces types les plus violents constituent également des échecs plus fréquents des tentatives $(302 \mathrm{C})$. Dans un cas comme dans l'autre, nous observons des sentences plus clémentes en ceci que plus diversifiées, moins conventionnelles, avec une durée d'emprisonnement plus courte. En fait, la variable dont la relation avec la sentence est la plus déterminante est l'ampleur de la dénonciation en termes de nombres d'événements reprochés et cela tient, en plus de la carrière réelle du voleur, de l'enquête policière qui va ou ne va pas mettre en lumière ces événements.

Le vol à main armée est un crime contre la personne; tout se passe cependant comme si, à l'intérieur de ce type d'infraction, il y avait ceux qui mettent en danger directement les personnes et ceux qui s'apparentent davantage à un crime contre les biens, l'acquisition d'argent. Curieusement par ailleurs, ce sont contre ces derniers que les réactions policières et judiciaires sont les plus impératives et les plus sévères. Les vols à main armée commis contre les institutions financières, outre la menace de l'arme, son! parmi ceux qui donnent le moins souvent lieu à un déploiement de violence; ils sont par ailleurs les plus payants. À l'autre extrémité, les vols à main armée commis contre les dépanneurs, commerces de variétés et les particuliers sont plus souvent le fait de suspects armés de couteau (autre arme offensive que l'arme à feu), où les contacts physiques avec les victimes et/ou témoins sont plus fréquents et où enfin le montant du vol est le plus bas. Ces vols à main armée pourraient par conséquent être davantage associés à un crime contre la personne.

Devant ces données statistiques, il est étonnant de noter que la réaction pénale concentre ses énergies sur les quelques délits, parmi cette catégorie de crimes dits violents, que l'on pourrait qualifier de "moins violents». Non seulement, sont-ils ceux qui présentent le moins souvent de violence réelle dans leur déroulement, mais ils sont aussi les moins nombreux dans l'ensemble des vols à main armée. Au niveau de la police de Montréal, une escouade spécialisée met toutes ses énergies dans la lutte contre de tels crimes et au niveau judiciaire l'adoption de l'article 83 montre clairement l'intention de réagir aux voleurs réalisant leur vol avec une arme à feu. Ceux qui s'attaquent aux banques sont ainsi armés, dans la presque totalité des cas, alors que ceux qui 
s'en prennent aux plus petits commerces et aux particuliers ont le plus souvent recours à une autre arme offensive. Par ailleurs, nous avons vu que dépendant du type de vol à main armée, les policiers et procureurs (concordance) ont recours à des étiquettes légales différentes bien que s'inscrivant généralement dans le cadre des articles 302 et 303 du code criminel. Cette constatation serait sans conséquence si nous ne constations parallèlement à cela des sentences différentes.

Ces quelques remarques ne sont pas faites dans le but de conclure à la nécessité de ne plus réagir avec célérité dans le cas de vols commis contre les institutions financières. Nous croyons cependant que l'effort pénal, s'il est juste de dire que le vol à main armée est un crime contre la personne, doit s'appliquer également, avec autant de fermeté vis-à-vis d'un crime que d'un autre. Si les voleurs qui s'en prennent aux institutions financières ont des sentences plus lourdes, c'est en partie dû au fait qu'ils se retrouvent devant le tribunal avec une dénonciation chargée, ce qui n'est pas le cas pour la plupart des autres vols. En d'autres termes, si l'enquête policière n'est pas la même pour tous les types de vol à main armée, si, dans la majorité des cas, l'enquête ne dépasse pas le vol à solutionner, on peut s'attendre à ce que les sentences imposées continuent de varier selon les types. Le résultat final d'une affaire dépend donc tant de la police que du tribunal.

L'ambiguité que nous observons dans la réaction des systèmes policier et pénal tient donc du caractère différentiel et inversé de cette réaction : les vols à main armée «objectivement» les moins violents donnent lieu à une réponse policière intense et à un traitement judiciaire plus rapide que les vols plus violents. Nous observons en quelque sorte une double scission entre la criminalité et la réaction policière d'une part et la criminalité et la réaction pénale d'autre part. Il ressort que les tribunaux réagissent largement en fonction du matériel recueilli par les policiers et dans ce sens ces deux instances sont en quelque sorte interdépendantes.

Les vols à main armée qui présentent une plus grande violence, c'est-à-dire les vols contre les dépanneurs, les commerces de variétés ainsi que les particuliers s'opposent aux vols moins violents notamment par le caractère des victimes, d'un côté des personnes et d'un autre essentiellement des institutions. Les vols à main armée de la première catégorie sont parmi les moins organisés, les moins bien exécutés, les moins payants, bref ils sont *socialement» moins importants d'autant plus que les com- 
merces visés ne font partie d'aucun regroupement particulièrement bien organisé et ayant pour raison d'être la défense des intérêts des «petits commerçants».

Ces observations nous amènent à penser qu'il serait souhaitable, pour corriger en quelque sorte cette ambiguité dans la réponse du système pénal, de chercher à mieux pondérer la VIOLENCE dans la sentence. Cet effort de pondération devrait nécessairement passer par l'appareil policier qui déploierait de plus grandes énergies pour les délits ayant suscité une plus grande violence et par les tribunaux qui pourraient réagir avec une plus grande similitude à tous les articles $302 \mathrm{~A}, \mathrm{~B}, \mathrm{C}$ ou D. Par ailleurs, une plus grande coopération entre instance policière et judiciaire conduirait peut-être à la démonstration plus fréquente des afameux articles 83», c'est-à-dire l'utilisation d'une arme à feu dans la commission d'une infraction.

Cet effort de pondération n'a pas de sens, croyons-nous, si nous ne parlons pas au même moment de la négociation de sentence. «Dans le contexte actuel, la sentence de l'avis de tous les agents de l'administration de la justice est définie par la négociation entre la couronne et la défense» (Rapport Laplante, p. 172). Que l'on soit d'accord ou non avec cette pratique, un fait est certain : l'enjeu consiste pour les deux parties à trouver, sur la base de la dénonciation et des éléments de preuve, le compromis qui permettra qu'il n'y ait pas de procès. Dans ce contexte, on peut se demander si l'esprit de la loi est respecté : le retrait fréquent de certains chefs permet de nous interroger sur la pertinence de poursuivre pour de tels chefs. Et si les corps policiers déployaient toutes leurs énergies à accumuler les preuves à fournir aux tribunaux, cela changerait-il quelque chose à ces pratiques? Un calcul rapide montre que l'individu ayant commis un vol à main armée a une sentence moyenne de 24,3 mois d'emprisonnement et celui qui a plus d'un événement, en moyenne 4,1 dans sa dénonciation, a une sentence moyenne de 39,4 mois d'emprisonnement, soit 9,6 mois par délit. La différence saute aux yeux. La négociation comporte donc, dans sa définition même, une forme de «clémence» pour les voleurs les plus actifs. Le législateur a-t-il vraiment à prévoir des sentences très sévères pour lutter contre le vol à main armée ou peut-on penser qu'une peine «raisonnable», mais appliquée autant de fois qu'il y a délits, ne pourrait pas amplement suffire pour réagir énergiquement contre cette forme de délit? Nous pensons que la proportion très élevée de plaidoyers de 
culpabilité s'explique notamment par la «garantie» d'une certaine clémence résultant de la négociation de la sentence. Beccaria écrivait : «La certitude d'une punition, même modérée, fera toujours plus d'impression que la crainte d'une peine terrible si à cette crainte se mêle l'espoir de l'impunité...» (Beccaria, p. 46) . Pour le voleur à main armée de carrière, il faudrait peut-être dire : ce n'est pas tant la certitude de la peine qui compte mais son adéquation avec le nombre de délits commis...'.

\section{RÉFÉRENCES}

BECCARIA, C. (1965), Des délits et des peines, Genève Droz, Édition Onzinode, 1787.

GAGNON, R., M. LEBLANC, (1984a), «Le vol à main armée dans les systèmes de justice», Recherche : le Vol à main armée, Rapport final no 3, Montréal, C.I.C.C./G.R.I.J., 357 p.

GAGNON, R., M. LEBLANC, (1984b), «L'intervention policière en matière de vol à main armée», lournal du collège canadien de police. 7,4 , pp. 317-330 (aussi en version anglaise).

GAGNON, R., M. LEBLANC, (1985), «Une typologie des vols à main armée à Montréal et à Québec», Revue canadienne de criminologie, $26,1$.

LAPLANTE, L. (1980), Rapport : le Vol à main armée au Québec, Québec ministère de la Justice, Direction des communications.

LEBLANC, M. (1985), «Le vol à main armée chez les mineurs», Criminologie, XVIII, 1.

9. Pour des détails supplémentaires concernant cette étude, voir le rapport : Gagnon, R. et LeBlanc, M. (1984), le Vol à main armée dans les systèmes de justice, G.R.I.J./C.I.C.C., Université de Montréal : 